\title{
Mitigating depolarizing noise on quantum computers with noise-estimation circuits
}

\author{
Miroslav Urbanek, ${ }^{1, *}$ Benjamin Nachman, ${ }^{2}$ Vincent R. Pascuzzi, ${ }^{2}$ \\ Andre He ${ }^{2, \dagger}$ Christian W. Bauer, ${ }^{2}$ and Wibe A. de Jong ${ }^{1, \ddagger}$ \\ ${ }^{1}$ Computational Research Division, Lawrence Berkeley National Laboratory, Berkeley, CA 94720, USA \\ ${ }^{2}$ Physics Division, Lawrence Berkeley National Laboratory, Berkeley, CA 94720, USA
}

\begin{abstract}
A significant problem for current quantum computers is noise. While there are many distinct noise channels, the depolarizing noise model often appropriately describes average noise for large circuits involving many qubits and gates. We present a method to mitigate the depolarizing noise by first estimating its rate with a noise-estimation circuit and then correcting the output of the target circuit using the estimated rate. The method is experimentally validated on the simulation of the Heisenberg model. We find that our approach in combination with readout-error correction, randomized compiling, and zero-noise extrapolation produces results close to exact results even for circuits containing hundreds of $C N O T$ gates.
\end{abstract}

\section{INTRODUCTION}

Noisy intermediate-scale quantum (NISQ) computers [1] are current and near-term quantum computers that are not fault-tolerant. The presence of noise and errors limits their utility. Even quantum algorithms designed for NISQ devices, for example the variational quantum eigensolver [2], are hampered by imperfections of real devices. Error rates are still too large to solve relevant scientific problems on existing quantum computers. Consequently, there has been a lot of effort to reduce noise and mitigate errors present on these devices.

An important class of errors are readout errors. They manifest themselves as readouts of incorrect qubit values during a measurement, e.g., reading one while the qubit is in the zero state and vice versa. Readout errors can be successfully mitigated with readout-error correction. Several method with varying degree of sophistication have been developed [3-8].

Another large source of errors are gate errors. They can be classified into coherent and incoherent errors. Coherent errors preserve state purity. They are typically small miscalibrations in control parameters. Coherent errors usually produce similar errors in consecutive executions of a quantum circuit and lead to a systematic bias in the output. Incoherent errors can be understood as either coherent errors with randomly varying control parameters or as processes that entangle the system with its environment. Incoherent errors are easier to handle than coherent errors, because they can often be modeled as depolarizing noise. A method for converting coherent errors into incoherent errors is randomized compiling [9-11].

Another practical technique of error mitigation is zeronoise extrapolation [12-22]. A circuit is executed multiple times with a varying degrees of noise and the measured output is extrapolated to the zero-noise limit. Other

\footnotetext{
* Corresponding author: urbanek@lbl.gov

$\dagger$ Present address: IBM Quantum, IBM T. J. Watson Research Center, Yorktown Heights, NY 10598 USA

$\ddagger$ Corresponding author: wadejong@lbl.gov
}

mitigation methods have been developed as well [23-35]. In this work, we introduce a new mitigation method. From a given quantum circuit, which we call a target circuit, we construct a circuit with a similar structure that we call an estimation circuit. We execute the estimation circuit to measure the depolarizing noise rate and then use the measured rate to correct the output of the target circuit. We experimentally demonstrate that the combination of readout-error correction, randomized compiling, mitigation with estimation circuits, and zero-noise extrapolation produces results that are very close to the exact results.

We first describe the method, introduce a simple class of estimation circuits, and present our full mitigation approach. We then show improvements obtained for our test case, which is a simulation of the Heisenberg model.

\section{METHODS}

A simple model of incoherent noise is the depolarizing noise model given by [36]

$$
\epsilon(\rho)=(1-p) \rho+p \frac{I}{2^{n}},
$$

where $\epsilon$ denotes the noise channel, $\rho$ is the density matrix, $p$ is the probabilistic error rate that depends on the device and also on the circuit, and $n$ is the number of qubits. Notice that if $\rho$ is initially a pure state, one can reconstruct the initial state from the noisy density matrix. For $p>0$, the initial pure state is the state with the largest weight in $\epsilon(\rho)$. Alternatively, if one knows $p$, the initial density matrix $\rho$ can be reconstructed simply by calculating the inverse $\epsilon^{-1}(\rho)$.

Observables are given by Hermitean operators acting on the system Hilbert space. They can be decomposed into sums of strings of identity and Pauli matrices,

$$
O=\sum_{i} c_{i} \prod_{j=1}^{n} \sigma^{i, j},
$$

where $c_{i}$ are real coefficients and $\sigma^{i, j} \in\left\{I, \sigma_{x}, \sigma_{y}, \sigma_{z}\right\}$ are identity or Pauli matrices acting on qubit $j$. The trace 
of $S=\prod_{j=1}^{n} \sigma^{i, j}$ is either $\operatorname{tr}(S)=2^{n}$ if $S$ is a product of identity matrices or $\operatorname{tr}(S)=0$ otherwise. The expectation value of an observable $O$ for a state represented by a density matrix $\rho$ is $\langle O\rangle=\operatorname{tr}(\rho O)$. The expectation value of $O$ for a noisy density matrix (1) is therefore given by

$$
\overline{\langle O\rangle}=\operatorname{tr}[\epsilon(\rho) O]=(1-p)\langle O\rangle+\frac{p}{2^{n}} \operatorname{tr}(O),
$$

where we denote the noisy expectation value by an overline. Notice that $\overline{\langle S\rangle}=1$ for strings $S$ consisting of identity matrices only and $\overline{\langle S\rangle}=(1-p)\langle S\rangle$ otherwise.

We can therefore decompose any observable $O$ as $O=$ $c I+O^{\prime}$, where $c$ is a constant, $I$ is the identity operator, and $\operatorname{tr}\left(O^{\prime}\right)=0$. Its expectation value is $\langle O\rangle=c+\left\langle O^{\prime}\right\rangle$. If we assume that the system decoherence is well described by the depolarizing noise model and if we know $p$, we can correct a noisy expectation value by calculating

$$
\langle O\rangle=\frac{\overline{\langle O\rangle}-c}{1-p}+c,
$$

where $\langle O\rangle$ is the corrected expectation value. We assume $c=0$ in the following without loss of generality, because $c$ is just a constant shift of the expectation value known in advance.

To correct the expectation value of any observable under the depolarizing noise model, we have to estimate the value of $p$. We do it by executing a circuit that is similar to our target circuit but has a known output. We assume that the target circuit consists of single-qubit and CNOT gates only and that CNOT gates are the leading source of gate errors. Our approach to construct an estimation circuit is to remove all single-qubit gates from the target circuit and to keep only the CNOT gates in it. Since the initial state on a quantum computer is the zero state, ideal CNOT gates do not transform the initial state at all. The final state is again a zero state on an ideal quantum computer. We can therefore estimate $1-p$ by measuring the probability of obtaining the zero state with the estimation circuit. The main assumption is the estimation and the target circuit are affected by a similar $p$ because they have the same structure.

It is not always necessary to remove all single-qubit gates. The estimation circuit can be any circuit that has a known output sensitive to noise and that has a similar structure as the target circuit. It may be beneficial to preserve some single-qubit gates to keep it similar to the target circuit. We add a layer of random rotations as the first circuit layer and its inverse as the last circuit layer to increase the robustness of the estimation.

Alternative recent approach uses near-Clifford circuits, which one can simulate classically, to perform mitigation [37]. The main difficulty of this approach is that the output of random near-Clifford circuits is similar to an output obtained with a completely mixed density matrix. One therefore has to select a particular subset of circuits that produce biased outputs. The authors used machine learning to find appropriate near-Clifford circuits with

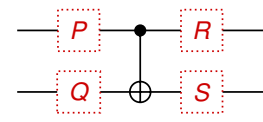

FIG. 1. Randomized compiling. Each $C N O T$ gate is dressed with the $P, Q, R$, and $S$ gates.

TABLE I. Gate choices in randomized compiling. Each $P$, $Q, R$, and $S$ assignment produces a dressed gate equal to a CNOT gate. An assignment is chosen independently and

\begin{tabular}{|c|c|c|c|}
\hline$P Q R S$ & $P Q R S$ & $\begin{array}{llll}P & Q & R & S \\
\end{array}$ & $P Q R S$ \\
\hline $\begin{array}{llll}I & I & I & I\end{array}$ & $\begin{array}{llll} & I & Y & X\end{array}$ & $\begin{array}{llll}X & I & X & X\end{array}$ & $\begin{array}{lll}Z & I & Z\end{array}$ \\
\hline $\begin{array}{llll}I & X & I & X\end{array}$ & $\begin{array}{llll}Y & X & Y & I\end{array}$ & $\begin{array}{llll}X & X & X & I\end{array}$ & $Z X Z X$ \\
\hline $\begin{array}{llll}I & Y & Z & Y\end{array}$ & $Y Y X$ & $\begin{array}{llll}X & Y & Y & Z\end{array}$ & $Z Y I$ \\
\hline$I \quad Z \quad Z \quad Z$ & $\begin{array}{llll}Y & Z & X & Y\end{array}$ & $\begin{array}{llll}X & Z & Y & Y\end{array}$ & $\begin{array}{llll}Z & Z & I & Z\end{array}$ \\
\hline
\end{tabular}
randomly and for each $C N O T$ gate in the circuit.

this property. Our method does not require any such selection. We simply remove single-qubit gates to obtain a biased circuit that can be simulated trivially.

We implemented our method in combination with readout-error correction, randomized compiling, and zeronoise extrapolation. Readout-error correction is performed using the unfolding $[8,38]$ method.

Coherent errors are dominant gate errors. They are not covered well by the depolarizing noise model. Randomized compiling [9] can convert coherent errors into incoherent errors. In particular, we consider single-qubit gates being the easy gates and CNOT gates being the hard gates. We perform randomized compiling by inserting a layer of randomizing single-qubit gates before and after each layer of CNOT gates as shown in Fig. 1. The randomizing gates are the identity and the Pauli gates. Each CNOT gate is preceded and succeeded by a pair of gates so that the overall action of the four single-qubit gates and a CNOT gate is exactly equal to a CNOT gate. All possible gate choices are listed in Table I. The layer of single-qubit gates after a layer of $C N O T$ gates can be composed with a layer of single-qubit gates before the next layer of $C N O T$ gates. The circuit structure therefore consists of layers of $C N O T$ gates interspersed with layers of single qubit gates. We use randomized compiling for both the estimation and the target circuit.

In the original formulation of zero-noise extrapolation $[13,16]$, the authors varied gate duration assuming that gate errors increase with longer gates. They ran experiments for several values of duration and extrapolated the measured results to zero duration. A pulse-level control is required to implement this method. This technique has been extended to systems with gate-level control [14]. The main assumption is that CNOT gates are the dominant source of errors. The authors replaced each CNOT gate with a sequence of three or five CNOT gates, which are equivalent to a single $C N O T$ gate, executed their circuits, and extrapolated to the zero-gate limit. This idea has been further extended to replace only a subset of 
CNOT gates with sequences of CNOT gates [18]. Both methods assume a certain dependence of errors on the number of $C N O T$ gates. In this work, we execute three versions of each circuit, where each CNOT gate is replaced by one, three, or five consecutive CNOT gates, and perform quadratic extrapolation to the limit corresponding to zero CNOT gates.

\section{EXPERIMENT}

Our test case is time evolution of the Heisenberg model. We consider a quench of a one-dimensional XX chain of noninteracting spin- $1 / 2$ particles [39]. Its Hamiltonian is given by

$$
H=-J \sum_{i=1}^{n-1}\left(\sigma_{x}^{j} \sigma_{x}^{j+1}+\sigma_{y}^{j} \sigma_{y}^{j+1}\right)
$$

where $J>0$ is a coupling constant, and $\sigma_{x}^{i}$ and $\sigma_{y}^{i}$ are Pauli matrices acting on qubit $j$. The system is initially prepared in a domain-wall configuration $\left|\psi_{0}\right\rangle=$ $|\ldots 111000 \ldots\rangle$ with qubits in the first and second half of the chain in the one and zero state, respectively. We consider $J=1$ and $\hbar=1$ in the following for simplicity.

The propagator $\exp (-i H t)$ is approximated by its second-order Trotter-Suzuki decomposition [40,41] to enable its implementation on a quantum computer. The approximated propagator for one time step is given by

$$
e^{-i H \Delta t} \approx e^{-i F \Delta t / 2} e^{-i G \Delta t} e^{-i F \Delta t / 2},
$$

where $F$ and $G$ contain terms in $H$ that act only on odd and even bonds, respectively, and $\Delta t$ is a time step. Since all terms in both $F$ and $G$ commute with each other, we can decompose the exponentials in Eq. (6) into products of two-qubit exponentials of the form $\exp \left[-i\left(\sigma_{x}^{j} \sigma_{x}^{j+1}+\right.\right.$ $\left.\left.\sigma_{y}^{j} \sigma_{y}^{j+1}\right) \Delta t / d\right]$, where $d \in\{1,2\}$. Each such exponential can be implemented by a circuit consisting of two CNOT gates and a number of single-qubit gates. One time step is therefore implemented by three layers of two-qubit circuits acting on odd, even, and odd bonds. Each twoqubit circuit is subsequently decomposed into two CNOT and multiple single-qubit gates. We measure the time evolution of the local magnetization of the last spin in the chain, $M_{n}(t)=\left\langle\psi(t)\left|\sigma_{z}^{n}\right| \psi(t)\right\rangle$.

We implemented this model on the IBM Q Paris device using six qubits Q23, Q24, Q25, Q22, Q19, and Q20 with 8192 shots for each circuit. The circuit is shown in Fig. 2. It contains 14 CNOT gates per time step. The longest circuit for 15 time steps contains 210 CNOT gates.

We created the estimation circuit from the target circuit by removing all its single-qubit gates. A layer of random single-qubit gates and a layer of their inverses were added as the first and the last circuit layer, respectively. Versions with one, three, and five $C N O T$ gates per each $C N O T$ gate were created. We then constructed and executed 448 randomized instances of each circuit. Readout errors were corrected using the unfolding technique as the first step in data processing. All expectation values were averaged over the randomized instances.

We estimated $1-p$ by measuring the $\left\langle\sigma_{z}^{6}\right\rangle$ expectation value with estimation circuits. Ideally, $\left\langle\sigma_{z}^{6}\right\rangle=1$, so the depolarizing noise rate is given by $1-p=\left\langle\sigma_{z}^{6}\right\rangle$. The mitigation was performed using Eq. (4). We then applied zero-noise extrapolation. Data points obtained with circuits with $n=1,3$, and $5 C N O T$ gates were extrapolated to $n=0$ using a quadratic fit. The final results are shown in Fig. 3.

The improvement obtained using the zero-noise extrapolation on top of the mitigation with estimation circuits can be understood as follows. An expectation value measured with circuits with artificially increased noise can be approximated by

$$
\overline{\langle O\rangle}_{n}=\left(1-p_{n}\right)\langle O\rangle \approx e^{-a n}\langle O\rangle,
$$

where $p_{n}$ is a noise rate, $\langle O\rangle$ is the exact expectation value, $a$ is a constant that depends on the circuit, and $n$ is a noise factor. In particular, $n=1,3$, and 5 for circuits with one, three, and five CNOT gates in place of each individual $C N O T$ gate. By estimating $1-p_{n}$ with the estimation circuit, we obtain a value that is close to the true $1-p_{n}$ for the target circuit, but not completely equal. However, the dependence on artificially increased noise has the same form as in (7),

$$
1-p_{n} \approx e^{-b n}
$$

where $b$ is a constant close to $a$. By performing mitigation with estimation circuits, we obtain

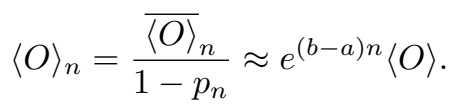

Since $|(b-a) n|$ is very small, we can approximate $\exp [(b-a) n] \approx 1+(b-a) n+(b-a)^{2} n^{2} / 2$. The quadratic extrapolation to $n=0$ then works well, because this quadratic function is a good approximation of the exponential function in the neighborhood of zero. In contrast, zero-noise extrapolation using expectation values obtained just with the target circuits does not work well because the quadratic function does not approximate the exponential well for large an. Another option may be to use an exponential function instead of a quadratic function to directly fit the data obtained with target circuits. We have found that this approach is very sensitive to noise and leads to worse results than the mitigation with estimation circuits. Fitting an exponential function may work better with less noisy data.

\section{CONCLUSION}

We presented a method to mitigate errors and noise on quantum computers that are described by the depolarizing noise model. The method prescribes a construction of an 
(a)

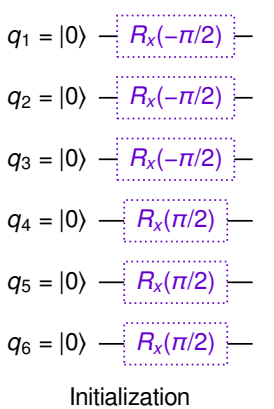

(b)

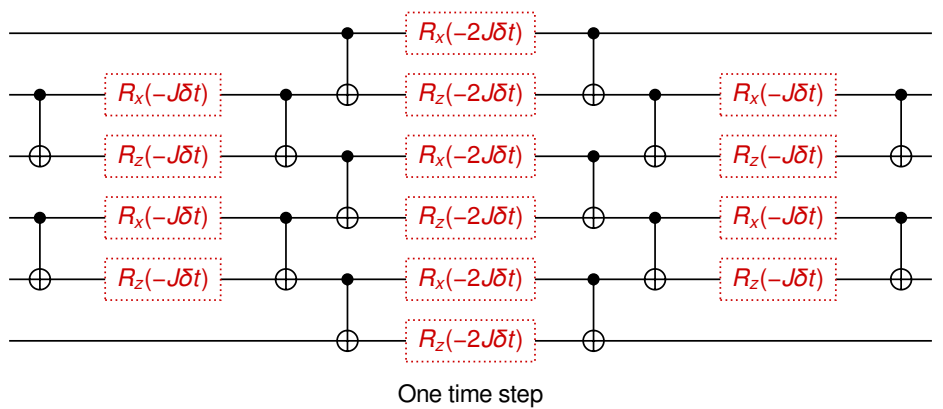

(c)

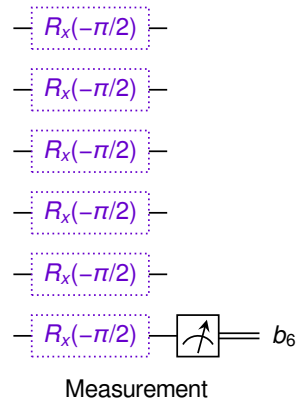

FIG. 2. Quantum circuit for the simulation of the XX chain. a) Preparation of the initial domain-wall state and basis transformation to a convenient basis. The dotted gates were replaced by random rotations in the estimation circuit. b) One step of the time evolution. Multiple steps are obtained by repeating this subcircuit. The dotted gates were removed in the estimation circuit. c) Basis transformation and measurement of the last qubit. The dotted gates were replaced by the inverses of random rotations from the initialization step in the estimation circuit.

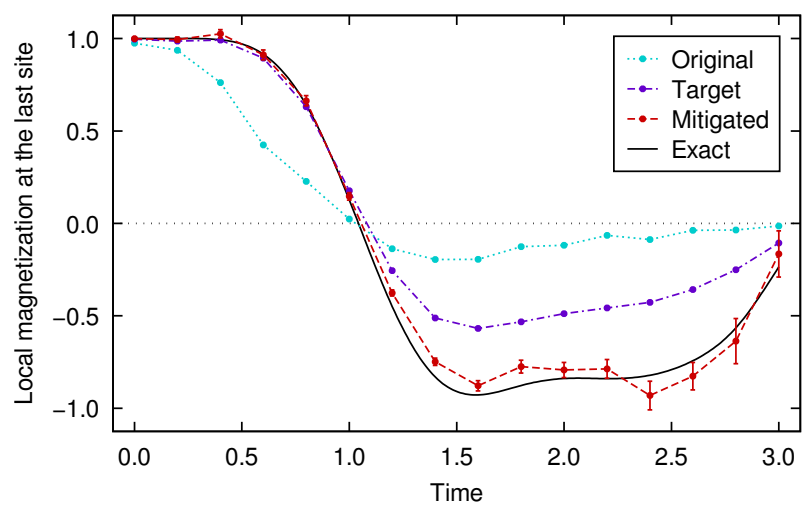

FIG. 3. Comparison of the original and mitigated results for the time evolution of the local magnetization in the XX chain. The original results were obtained using the original circuits without any mitigation. There are $14 C N O T$ gates per time step and the longest original circuit contains 210 CNOT gates. Target results use readout-error correction, randomized compiling, and zero-noise extrapolation. Mitigated results use readout-error correction, randomized compiling, mitigation with estimation circuits, and zero-noise extrapolation. Data for extrapolation were obtained with circuits where each $C N O T$ gate was replaced by one, three, or five CNOT gates. Each circuit was executed with 448 random instances. Error bars represent the standard deviation of processed data. Exact solution takes the Trotter-Suzuki decomposition into account.

estimation circuit to estimate the noise rate that is then used to correct the output of a given circuit. A crucial part of this approach is the randomized compiling that ensures that gate errors can be modeled as incoherent depolarizing noise. We demonstrated that the method works well, especially in combination with readout-error correction and zero-noise extrapolation, on a set of test circuits containing hundreds of CNOT gates. The method is scalable to any number of qubits and gates given that enough randomized samples are collected to achieve low uncertainty.

\section{ACKNOWLEDGMENTS}

This work was supported by the U.S. Department of Energy (DOE) under Contract No. DE-AC02-05CH11231, through the Office of Advanced Scientific Computing Research Quantum Algorithms Team Program, and the Office of High Energy Physics through the Quantum Information Science Enabled Discovery program (Grant No. KA2401032). This research used resources of the Oak Ridge Leadership Computing Facility, which is a DOE Office of Science User Facility supported under Contract No. DE-AC05-00OR22725.

\section{Appendix A: Zero-noise extrapolation}

We replaced each CNOT gate in our circuits with one, three, or five consecutive CNOT gates to artificially increase noise. They are equivalent to a single $C N O T$ gate on a noiseless quantum computer. Both the estimation and the target circuits were modified. Figure 4 shows the effect of extra CNOT gates on the output of the estimation circuits. Figure 5 shows this effect on the output of the target circuits. We performed mitigation using the outputs of the estimation and target circuits to obtain mitigated local magnetization shown in Fig. 6 . 
Nat. Commun. 5, 4213 (2014).

[3] A. Kandala, A. Mezzacapo, K. Temme, M. Takita, M. Brink, J. M. Chow, and J. M. Gambetta, Nature 549, 242 (2017).

[4] F. B. Maciejewski, Z. Zimborás, and M. Oszmaniec, Quantum 4, 257 (2020).

[5] S. Bravyi, S. Sheldon, A. Kandala, D. C. Mckay, and J. M. Gambetta, Mitigating measurement errors in multi-qubit experiments, arXiv (2020), arXiv:2006.14044 [quant-ph].

[6] L. Funcke, T. Hartung, K. Jansen, S. Kühn, P. Stornati, and X. Wang, Measurement error mitigation in quantum computers through classical bit-flip correction, arXiv (2020), arXiv:2007.03663 [quant-ph].

[7] J. W. O. Garmon, R. C. Pooser, and E. F. Dumitrescu, Phys. Rev. A 101, 042308 (2020).

[8] B. Nachman, M. Urbanek, W. A. de Jong, and C. W. Bauer, npj Quantum Inf. 6, 84 (2020).

[9] J. J. Wallman and J. Emerson, Phys. Rev. A 94, 052325 (2016).

[10] Z. Cai and S. C. Benjamin, Sci. Rep. 9, 11281 (2019).

[11] Z. Cai, X. Xu, and S. C. Benjamin, npj Quantum Inf. 6, 17 (2020).

[12] Y. Li and S. C. Benjamin, Phys. Rev. X 7, 021050 (2017).

[13] K. Temme, S. Bravyi, and J. M. Gambetta, Phys. Rev. Lett. 119, 180509 (2017).

[14] E. F. Dumitrescu, A. J. McCaskey, G. Hagen, G. R. Jansen, T. D. Morris, T. Papenbrock, R. C. Pooser, D. J. Dean, and P. Lougovski, Phys. Rev. Lett. 120, 210501 (2018).

[15] S. Endo, S. C. Benjamin, and Y. Li, Phys. Rev. X 8, 031027 (2018).

[16] A. Kandala, K. Temme, A. D. Córcoles, A. Mezzacapo, J. M. Chow, and J. M. Gambetta, Nature 567, 491 (2019).

[17] S. McArdle, X. Yuan, and S. Benjamin, Phys. Rev. Lett. 122, 180501 (2019).

[18] A. He, B. Nachman, W. A. de Jong, and C. W. Bauer, Phys. Rev. A 102, 012426 (2020).

[19] J. Sun, X. Yuan, T. Tsunoda, V. Vedral, S. C. Bejamin, and S. Endo, Practical quantum error mitigation for analog quantum simulation, arXiv (2020), arXiv:2001.04891 [quant-ph].

[20] F. G. Fuchs, V. Falch, and C. Johnsen, Eur. Phys. J. Plus 135, 353 (2020).

[21] A. Strikis, D. Qin, Y. Chen, S. C. Benjamin, and Y. Li, Learning-based quantum error mitigation, arXiv (2020), arXiv:2005.07601 [quant-ph].

[22] S. Tomkins and R. de Sousa, Noise mitigation with delay pulses in the ibm quantum experience, arXiv (2020), arXiv:2005.12520 [quant-ph].

[23] X. Bonet-Monroig, R. Sagastizabal, M. Singh, and T. E. O'Brien, Phys. Rev. A 98, 062339 (2018).

[24] I. Schwenk, J.-M. Reiner, S. Zanker, L. Tian, J. Leppäkangas, and M. Marthaler, Phys. Rev. A 97, 042310 (2018).

[25] J.-M. Reiner, S. Zanker, I. Schwenk, J. Leppäkangas, F. Wilhelm-Mauch, G. Schön, and M. Marthaler, Quantum Sci. Technol. 3, 045008 (2018).

[26] S. Endo, Q. Zhao, Y. Li, S. Benjamin, and X. Yuan, Phys. Rev. A 99, 012334 (2019).

[27] M. Otten and S. K. Gray, Phys. Rev. A 99, 012338 (2019).

[28] M. Otten and S. K. Gray, npj Quantum Inf. 5, 11 (2019).

[29] M. Otten, C. L. Cortes, and S. K. Gray, Noise-resilient quantum dynamics using symmetry-preserving ansatzes, arXiv (2019), arXiv:1910.06284 [quant-ph].
[30] D. C. Murphy and K. R. Brown, Phys. Rev. A 99, 032318 (2019).

[31] R. Sagastizabal, X. Bonet-Monroig, M. Singh, M. A. Rol, C. C. Bultink, X. Fu, C. H. Price, V. P. Ostroukh, N. Muthusubramanian, A. Bruno, M. Beekman, N. Haider, T. E. O'Brien, and L. DiCarlo, Phys. Rev. A 100, 010302 (2019).

[32] S. E. Smart and D. A. Mazziotti, Phys. Rev. A 100, 022517 (2019).

[33] C. Song, J. Cui, H. Wang, J. Hao, H. Feng, and Y. Li, Sci. Adv. 5, eaaw5686 (2019).

[34] S. Zhang, Y. Lu, K. Zhang, W. Chen, Y. Li, and K. Zhang, Jing-Ningand Kim, Nat. Commun. 11, 587 (2020).

[35] A. Roggero and A. Baroni, Phys. Rev. A 101, 022328 (2020).

[36] M. A. Nielsen and I. L. Chuang, Quantum Computation and Quantum Information: 10th Anniversary Edition (Cambridge University Press, Cambridge, 2010).

[37] P. Czarnik, A. Arrasmith, P. J. Coles, and L. Cincio, Error mitigation with clifford quantum-circuit data (2021), arXiv:2005.10189 [quant-ph].

[38] M. Urbanek, B. Nachman, and W. A. de Jong, Phys. Rev. A 102, 022427 (2020).

[39] A. Smith, M. S. Kim, F. Pollmann, and J. Knolle, npj Quantum Inf. 5, 106 (2019).

[40] H. F. Trotter, Proc. Am. Math. Soc. 10, 545 (1959).

[41] M. Suzuki, Commun. Math. Phys. 51, 183 (1976). 


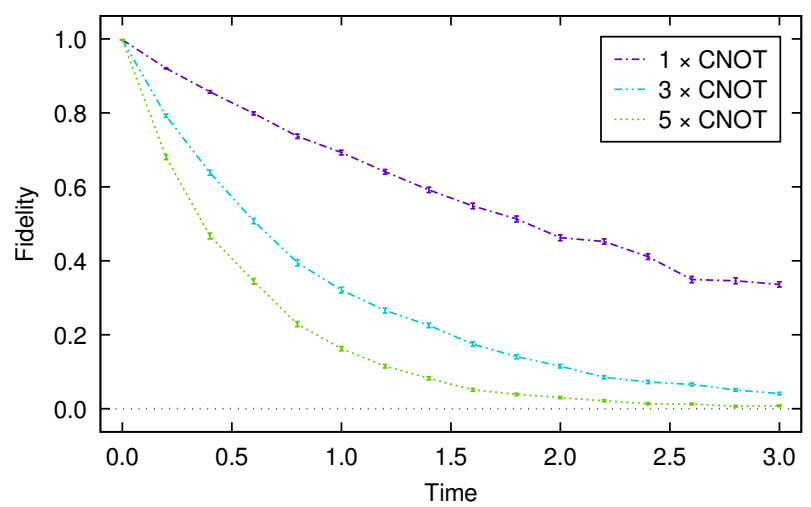

FIG. 4. Fidelity $1-p$ measured with the estimation circuits. Each point is a mean of values obtained by executing 448 randomized circuit instances. Error bars represent the standard error of the mean. Readout error correction was applied to measured data.

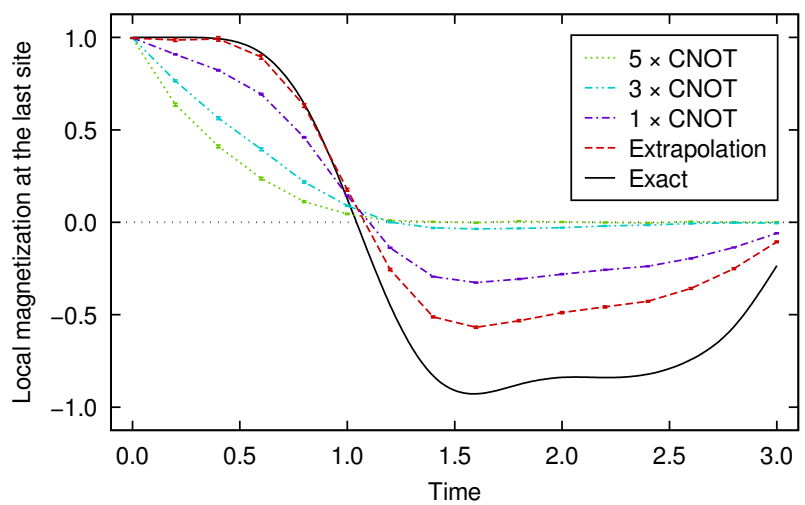

FIG. 5. Local magnetization measured with the target circuits. Each point is a mean of values obtained by executing 448 randomized circuit instances. Error bars represent the standard error of the mean. Readout error correction was applied to measured data. Local magnetization was extrapolated to the zero-noise limit. 


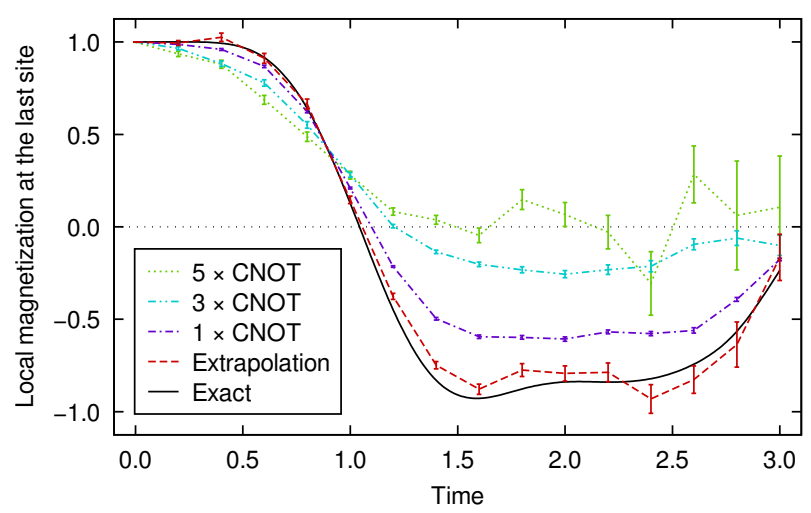

FIG. 6. Mitigated local magnetization obtained from the the outputs of the estimation and target circuits. Error bars represent the standard deviation. Local magnetization was extrapolated to the zero-noise limit. 